\title{
Enhanced Fireworks Algorithm-Auto Disturbance Rejection Control Algorithm for Robot Fish Path Tracking
}

\author{
X. Song, S. Gao, C. Chen, Z. Gao
}

\author{
Xiaoru Song \\ School of Electronic and Information Engineering, Xian Technological University \\ No.2 of Xuefu Middle Road, Xian, Shaanxi, China \\ masha0422@163.com \\ Song Gao* \\ School of Electronic and Information Engineering, Xian Technological University \\ No.2 of Xuefu Middle Road, Xian, Shaanxi, China \\ *Corresponding author: gao2223@qq.com

\section{Chaobo Chen} \\ School of Electronic and Information Engineering, Xian Technological University \\ No.2 of Xuefu Middle Road, Xian, Shaanxi, China \\ 17777108@qq.com

\section{Zepeng Gao} \\ School of Electronic and Information Engineering, Xian Technological University \\ No.2 of Xuefu Middle Road, Xian, Shaanxi, China \\ 1067456190@qq.com
}

\begin{abstract}
The robot fish is affected by many unknown internal and external interference factors when it performs path tracking in unknown waters. It was proposed that a path tracking method based on the EFWA-ADRC (enhanced fireworks algorithmauto disturbance rejection control) to obtain high-quality tracking effect. ADRC has strong adaptability and robustness. It is an effective method to solve the control problems of nonlinearity, uncertainty, strong interference, strong coupling and large time lag. For the optimization of parameters in ADRC, the enhanced fireworks algorithm (EFWA) is used for online adjustment. It is to improve the anti-interference of the robot fish in the path tracking process. The multi-joint bionic robot fish was taken as the research object in the paper. It was established a path tracking error model in the Serret-Frenet coordinate system combining the mathematical model of robotic fish. It was focused on the forward speed and steering speed control rate. It was constructed that the EFWA-ADRC based path tracking system. Finally, the simulation and experimental results show that the control method based on EFWA$\mathrm{ADRC}$ and conventional ADRC makes the robotic fish track the given path at $2.8 \mathrm{~s}$ and $3.3 \mathrm{~s}$ respectively, and the tracking error is kept within plus or minus $0.09 \mathrm{~m}$ and $0.1 \mathrm{~m}$ respectively. The new control method tracking steady-state error was reduces by $10 \%$ compared with the conventional ADRC. It was proved that the proposed EFWA-ADRC controller has better control effect on the controlled system, which is subject to strong interference.

Keywords: Active disturbance rejection control, robot fish, path tracking, enhanced fireworks algorithm.
\end{abstract}

\section{Introduction}

The use of bionic robot fish is deepened in narrow or dangerous underwater environments, such as monitoring, underwater archaeology, underwater equipment overhaul, military investigation, etc. The new challenges are imposed on the intelligent requirements of robot fish. The path tracking is involved the kinematics and complex hydrodynamic characteristics of the bionic robot 
fish body in the unknown waters, as well as coupling, system nonlinearity, and the influence of various unknown internal and external disturbance factors. Many domestic and overseas scholars have conducted a lot of research on the tracking of robotic fish, and have achieved certain results. The path tracking only requires the robot fish to converge to the desired path. The desired position is not constrained by the time condition. It can make the robot fish complete the path tracking task at the desired speed, so it can smoothly converge to the desired path, and the control input saturation is not easy to occur. Therefore, it is beneficial to improve the endurance of the robot fish. However, the design of the controller becomes more difficult due to external disturbances, limited lateral and vertical motion limitations, high nonlinearity and uncertainty of hydrodynamic coefficients. The literature $[7,25]$ establishes the horizontal plane tracking error equation based on the virtual wizard, and then designs the forward speed controller based on Lyapunov theory and backstepping method to realize the path tracking of the horizontal plane. In [26], the tracking error equation is established by introducing the approach angle and virtual guide, and then the horizontal path tracking controller is designed based on Lyapunov theory and backstepping method. Phi Luan proposed a robotic fish dynamics model with a non-uniform flexible tail (NFT). It was studied the relationship between input torque and speed. The dynamic model of the robotic fish was verified through experiments. But the problem has not been resolved the motion control problem of the robotic fish [1]. A point-and-point stabilization algorithm was proposed for robotic fish. This strategy aims to eliminate the direction error and distance error between the starting point and the target position of the robotic fish. It is not considered the real-time position control of the robotic fish during the tracking process. In addition, the model is not accurate enough and complicated water wave interference. The actual control effect is not ideal. A fuzzy PID control method was proposed. It was optimized the mathematical model of the robot fish. The robot deep-motion tracking was completed by the fuzzy self-tuning method. The robotic fish has good anti-interference during the tracking process. And it does not require an accurate mathematical model of the robotic fish. It can be effectively implemented by the human experience and control strategies. However, the fuzzy control setting rules require expert knowledge or a large number of experiments to obtain. It cannot be widely applied to the control of the robot fish [9]. A sliding-mode path tracking was proposed [11]. It is well adapted to the nonlinear, indeterminate state of the model. The simulation results show it has better control accuracy. However, it is prone to chattering effect due to inertia and time lag factors. A new method was proposed a curve path tracking based on line-of-sight navigation by Signe Moe considering the interference of the ocean current environment. The line guidance law and the sliding mode adaptive feedback linearization controller were used for path tracking to obtain good control effects. However, the chattering problem has not been solved [4]. The ADRC control method was proposed for the fish-like finfish. The simulation shows that the method has good dynamic and static characteristics for the fish heading control [7]. It was proposed a path tracking based on ADRC under-actuated AUV. The simulation results show that the chattering phenomenon and overshoot caused by interference is well suppressed [6]. And the ADRC parameters are set according to experience in the paper. It may not be optimal $[14,15]$.

There are many ADRC parameter tuning methods. The bandwidth-based linearized ADRC was proposed by Gao Z. The good effect of this method is obtained in practical applications. However, this method does not give full play to the advantages of nonlinear mechanism [25]. The steady-state performance and rapidity of the control system are considered comprehensively based on the artificial intelligence-based parameter tuning method. The artificial immune algorithm and particle swarm optimization algorithm were used to adjust the ADRC parameters [5]. It has the disadvantages of time-consuming and inevitable local minimum. The chaotic particle swarm optimization algorithm was to adjust the parameters of the controller [8]. But large time consuming is still exists. The reinforcement learning was proposed to adaptive the ADRC algo- 
rithm parameters. It has a long online learning time. the continuous state space is not easy to be processed. When the state is large, the dimension explosion problem is easy to appear [19]. The fireworks algorithm was proposed by Chinese scholar Tan Ying. The algorithm has good performance and high efficiency in solving complex optimization problems [18].

In summary, the enhanced fireworks algorithm is adopted to optimize the parameters of ADRC. And a path tracking algorithm based on EFWA-ADRC is proposed to make the highquality tracking effect of the robot fish in the path tracking process. The multi-joint bionic robot fish is used as the research object in the paper. The path tracking error equation is established in the SF coordinate system. The expected angle guidance algorithm is introduced to design the required heading angle and the guidance rate of the forward speed control to eliminate the tracking error. It is proved the stability of the error dynamics equation. On this basis, the EFWA-ADRC controller is used to control the forward speed and yaw angle of the robot fish respectively. So the robot fish can track the given path at the desired speed comparing with the conventional ADRC control method to verify the feasibility and reliability of the proposed controller $[16,17,20]$. The rest of the paper is organized as follows. The problem description is presented in the section 1. The part 2 is the key. It is designed of EFWA-ADRC second-order auto-disturbance path tracking controller. The simulation and experiment of the tracking controller of the EFWA-ADRC algorithm are in the section 3. At last, conclusions are drawn [21,22].

\section{Problem description}

\subsection{Multi-joint robotic fish mathematical model}

The multi-joint bionic robot is a special underwater robot. It mimics the way in which hundreds of millions of years of fish have evolved. The propulsion model of the robotic fish is proposed by the literature [2]. The four-joint robotic fish is used as the research object in the paper. It consists of one body link, two movable tail links and a passive caudal fin link. That is four links to approximate the state of fish movement at each moment. Fig.1 is a four-joint robotic fish structure. The position of the robot fish each link is obtained by digital fitting method [13].

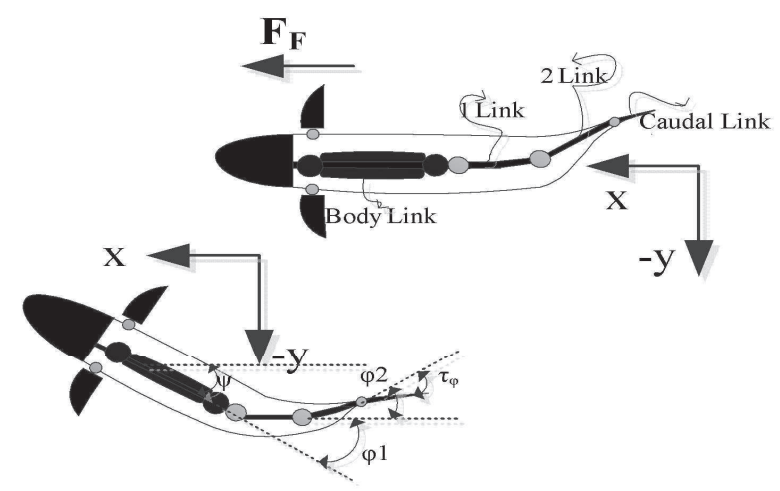

Figure 1: The structure diagram of the robot fish

There are strong coupling between the connecting rods of the robot fish in practical applications. It forces the robot fish analysis is very complicated due to the limitation of the actuator and mechanical structure. The thrust most generated by the caudal fin contributes during the movement of the robotic fish. 
In order to simplify the research, the two joints force analysis of the tail is mainly carried out, and the dynamic equation of the robot fish is obtained as follows (1).

$$
\left\{\begin{array}{r}
M_{R H} \dot{v}+C_{R B}(v) v=\tau_{H} \\
\tau_{H}=-C_{A}\left(v_{r}\right) v_{r}-D\left(v_{r}\right) v-g(\Theta)+A_{c}\left(v_{c}\right) v_{r}+\tau_{C}
\end{array}\right.
$$

where, $\tau_{H}$ is the thrust of the machine fish to get forward. $V_{r}$ is the speed of the robot fish relative to the water flow. $V_{c}$ is the speed of the water flow. $C_{R B}(v)$ is the fish body coefficient, $C_{A}\left(v_{r}\right)$ is the water flow force matrix. $D\left(v_{r}\right)$ is the total water flow damping matrix. $g(\Theta)$ is a vector matrix containing gravity and buoyancy. $A_{c}\left(v_{c}\right)$ is the ocean current velocity Gaussian Markov process thrust, and $M_{R H}$ is the total mass of the robotic fish. $M_{R H}=M_{R B}+M_{A}, M_{R}$ is the mass matrix of the rigid body, and $M_{A}$ is the additional mass matrix of the fish body. The fine derivation process can be referred to the literature [10].

\subsection{Robotic path tracking error model in carrier coordinate system}

The robotic fish movement is more complicated. In order to simplify the research, it is assumed that the robotic fish only moves in the horizontal plane, and ignores the robotic yaw, roll, and pitch motion. The following three coordinate systems are used, as shown in Fig.2 in the paper. They are the inertial coordinate system $E-\xi \eta\{I\}$ of the robot fish, the fish body coordinate system $O-x y\{B\}$, and the SF coordinate system $P-x_{p} y_{p}\{S F\}$. Where, Point $P$ on the $S F$ coordinate system is a free reference point on the reference path, expressed as a virtual target robot. $P-x_{p(s)}$ is the tangent direction of the reference path. $P-y_{p(s)}$ is the normal direction of the reference path. The speed vector of the robot fish is $V_{t}$, the speed of each component in the coordinate system of the fish body is expressed as $(u, v, r)$, where $(u, v, r)$ is forward speed. lateral speed, steering speed respectively, where $\psi$ is the angle between $O-x$ and the positive half of the $E-\xi$. Assume that the reference point $P$ (virtual robot) moves along the reference path at speed $U_{P}$. The angle between the $P-x_{p(s)}$ axis and the $E-\xi \eta\{I\}$ axis of the ground coordinate system is $\psi_{p}$.

The kinematic equation of the robot fish is as follows formula (2)

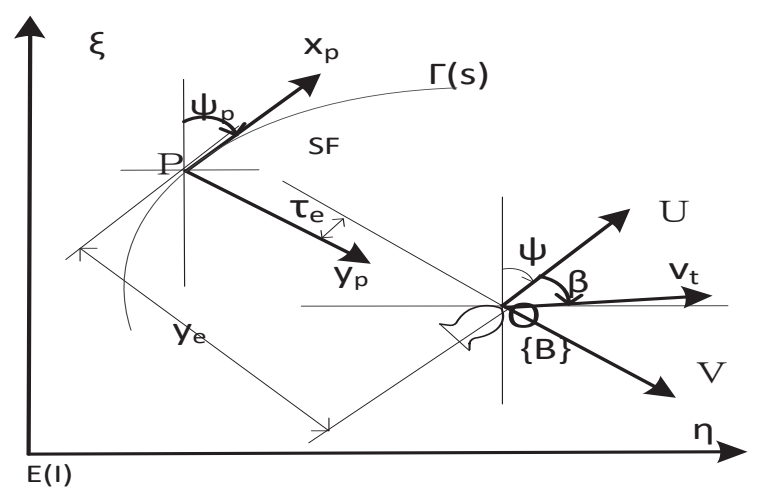

Figure 2: The schematic diagram of robot fish path tracking

$$
\left\{\begin{array}{r}
\dot{\xi}=\cos \psi \cdot u-\sin \psi \cdot v \\
\dot{\eta}=\sin \psi \cdot u+\cos \psi \cdot v \\
\dot{\psi}_{\mathrm{B}}=\mathrm{r}+\dot{\beta}
\end{array}\right.
$$

In the formula $(2) \psi_{B}$ is the angle between the synthetic fish speed $V_{t}$ and the $E-\xi$. $\beta$ is the drift angle of the robot fish. It is $\psi=\psi_{B}+\beta$. To simplify the study, let $\beta=\arctan (v / u)$. 
The reference path is described by curve $\Gamma(s)$. The reference point $P\left(x_{p(s)}, y_{p(s)}\right)$ on the curve is uniquely determined by the $\Gamma(s)$ curve. In order to calculate the error angle, the distance from the centroid point $O$ of the robot fish to the $P$ point of the virtual robot is projected to the $S F$ coordinate system. The error dynamics system $\left(\tau_{e}, y_{e}, n_{e}\right)$ under the coordinate system is obtained, that is the equation (3) is the path following error dynamics equation.

$$
\left\{\begin{array}{r}
\dot{\tau}_{e}=\dot{s}-y_{e} w_{p}-v_{t} \cos \psi_{e} \\
\dot{y}_{e}=\tau_{e} w_{p}-v_{t} \sin \psi_{e} \\
\dot{\psi}_{e}=w_{p}-r-\dot{\beta}
\end{array}\right.
$$

\section{Design of EFWA-ADRC second-order auto-disturbance path tracking controller}

\subsection{Kinematic control rate design}

Forward speed is used to control the actual speed $V_{t}$. the goal is the forward speed $u$ is maintained at a constant value $u_{d}$. That is constant greater than zero throughout the tracking process.

Define the tracking speed error as:

$$
u_{e}=u-u_{\mathrm{d}}
$$

The elimination of the tangential error $\tau_{e}$ needs to be controlled by selecting an appropriate $U_{p}$ such that the tangential error $\tau_{e}$ approaches zero. For the normal error, it needs to be controlled by the angle $\psi$. The method of the desired angle is employed in the paper, where in the desired angle $\psi_{d}\left(y_{e}\right)$ satisfies the requirement of the following formula (5)

$$
\left\{\begin{array}{c}
\psi_{d}(0)=0 \\
y_{e} \psi_{d}(e) \geq 0, \forall y_{e} \in R
\end{array}\right.
$$

The expression of the desired angle $\psi_{d}\left(y_{e}\right)$ selected is the formula (6).

$$
\psi_{d}=\arctan \left(k_{e} y_{e}\right), \quad \psi_{d} \in\left(-\frac{\pi}{2}, \frac{\pi}{2}\right)
$$

In equation (6), $k_{e}>0$ is the normal tracking control parameter. It can be found from the above equation error dynamics equation that as long as the attitude tracking $\psi_{e}$ angle converges to $\psi_{d}$ and $x_{e}$ converges to zero, then $y_{e}$ naturally converges to zero, then it can be known that the approach angle $\psi_{d}$ converges to zero.

Therefore, the control rate of the following formula (7) is selected in this paper. The speed of the reference point $\mathrm{P}$ is:

$$
\left\{\begin{array}{c}
U_{p}=U_{d}+k_{\tau} \tau_{e} \\
r_{d}=w_{p}-\dot{\beta}-\dot{\psi}_{d}+k_{1}\left(\psi_{e}-\psi_{d}\right) \\
\dot{s}=k_{\tau} \tau_{e}+v_{t} \cos \psi_{e}
\end{array}\right.
$$

Define the Lyapunov function as shown in the following equation (8)

$$
V_{1}=\frac{1}{2}\left(\psi_{e}-\psi_{d}\right)^{2}
$$

Its derivative is equation (9): 


$$
\begin{aligned}
& \dot{V}_{1}=\left(\psi_{e}-\psi_{d}\right)\left(\dot{\psi}_{e}-\dot{\psi}_{d}\right)=\left(\psi_{e}-\psi_{d}\right)\left(w_{p}-r-\dot{\beta}-\dot{\psi}_{d}\right) \\
& =-k_{1}\left(\psi_{e}-\psi_{d}\right)^{2} \leq 0
\end{aligned}
$$

In the formula $(9), k_{1}$ is a number greater than zero. So $V_{1}$ is a monotonic non-increasing function, so function $V_{1}$ is bounded. Since $V_{1}$ has consistent continuity, it can be obtained according to Barbala's lemma:

$$
\lim _{t \rightarrow \infty} \dot{V}_{1}=0 \Rightarrow \lim _{t \rightarrow \infty} \psi_{e}=\lim _{t \rightarrow \infty} \psi_{d}
$$

Define the second Lyapunov function as shown in the following equation (11):

$$
V_{2}=\frac{1}{2}\left(\tau_{e}^{2}+y_{e}^{2}\right)
$$

Derived for it, its derivative function is equation (12)

$$
\begin{aligned}
& \dot{V}_{2}=\tau_{e} \dot{\tau}_{e}+y_{e} \dot{y}_{e}=\tau_{e}\left(\dot{s}-v_{t} \cos \left(\psi_{e}\right)\right)-y_{e} v_{t} \sin \left(\psi_{e}\right) \\
& =-k_{2} \tau_{e}{ }^{2}-y_{e} v_{t} \sin \left(\psi_{e}\right)
\end{aligned}
$$

Because $y_{e} \psi_{d}\left(y_{e}\right) \geq 0, V_{t} \geq 0$ and $y_{e} v_{t} \sin \left(\psi_{e}\right) \geq 0$, then $V_{2}$ is a monotonic non-increasing function. According to Barbala's lemma, it can be seen that $x_{e}, y_{e}, \psi_{e}$ will converge to zero under the control rate of equation (7), so the robot fish will converge to the desired path.

\subsection{Enhanced fireworks algorithm}

The enhanced fireworks algorithm (EFWA) consists of an explosion operator, a mutation operator, a mapping rule, and a selection strategy. The flow chart of the enhanced fireworks algorithm is shown in Fig.3.

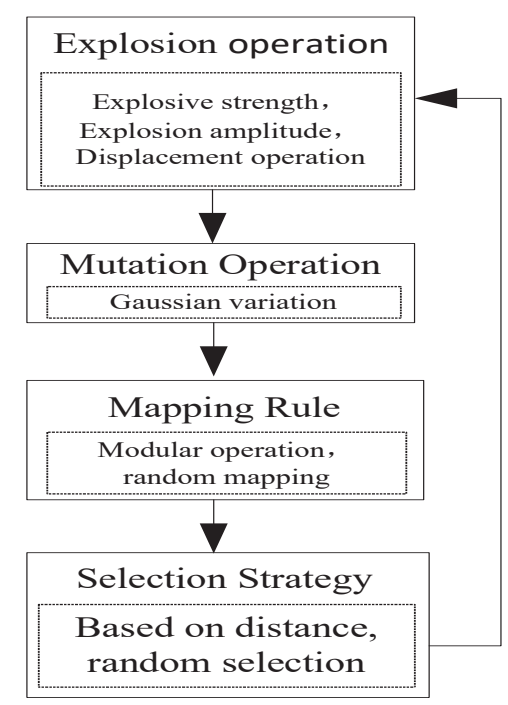

Figure 3: The flow chart of enhanced fireworks algorithm

(1) Explosion operator

$$
S_{i}=S N \cdot \frac{y_{\max }-f_{i}+\varepsilon}{\sum_{i=1}^{N}\left(y_{\max }-f_{i}\right)+\varepsilon}
$$


$N$ is the total number of initial fireworks per generation in equation (13). $S_{i}$ is the spark generated by each fireworks explosion. $S N$ is the total number of explosion sparks. $f_{i}$ is the individual fitness value, and $y_{m} a x$ is the current population fitness value. $S_{i}$ is a small number. The fireworks $S_{i}$ have the following controls:

$$
S_{i}=\left\{\begin{array}{c}
\operatorname{round}(a \cdot S N), S_{i}<a \cdot S N \\
\operatorname{round}(b \cdot S N), S_{i}>b \cdot S N \\
\operatorname{round}\left(S_{i}\right), \text { others }
\end{array}\right.
$$

$a, b$ is a constant, and round is a rounding function. The following formula (15) is the magnitude of the explosion:

$$
A_{i}=A \cdot \frac{f_{i}-y_{\min }+\varepsilon}{\sum_{i=1}^{N}\left(f_{i}-y_{\min }\right)+\varepsilon}
$$

$A$ is the maximum explosion amplitude of fireworks, and $y_{m} i n$ is the optimal fitness value of the current population. $A_{\min , k}$ is the detection threshold with the lowest explosion radius in the $k$ th dimension, that is:

$$
A_{i k}=\left\{\begin{array}{c}
A_{\min , k} A_{i k}<A_{\min , k} \\
A_{i k} \text { others }
\end{array}\right.
$$

where, $t$ is the number of evaluations for the current iteration, evals $s_{m} a x$ is the maximum number of evaluations, $A_{\text {init }}$ and $A_{\text {final }}$ are the initial and final blast radius detection values.

$$
A_{\min , k}(t)=A_{\text {init }}-\frac{A_{\text {init }}-A_{\text {final }}}{\text { evals }} \sqrt{\left(2 e v a l s_{\max }-t\right) t}
$$

(2) Displacement operation and mapping rules The $S_{i}$ explosion sparks are position offset according to equation (18) and then an explosion spark is generated.

$$
\mathrm{X}_{i k}=X_{i k}+A_{i} \cdot U(-1,1)
$$

$U(-1,1)$ is a uniform distribution between intervals $[-1,1]$. When the spark $x_{i}$ exceeds the boundary on the kth dimension, it will be out of bounds detection by the mapping rule of equation (19), and mapped to a new position.

$$
X_{i k}=X_{L B, k}+U(0,1) \times\left(X_{U B, k}-X_{L B, k}\right)
$$

In equation (20), $X_{L B, k}, X_{U B, k}$ are the solution space at the lower and upper boundaries of the $k t h$ dimension, and $U(0,1)$ is a uniformly distributed random number over the $[0,1]$ interval.

(3) Gaussian variation The number $N$ fireworks are randomly selected in $k t h$ dimensions, and each firework is subjected to Gaussian mutation operation in each dimension according to equation (20).

$$
X_{i k}=X_{i, k}+\left(X_{B, k}-x_{i k}\right) N(0,1)
$$

where, $N(0,1)$ is a Gaussian distribution.

(4) Random selection strategy In order to ensure that the good individuals can be passed to the next generation in the iterative process, the optimal individual is first retained. And the remaining $N-1$ fireworks are selected according to the random selection. 


\subsection{Design of EFWA-ADRC second-order auto-disturbance path tracking controller}

It can be seen that each component of ADRC realizes functions independently by analyzing the principle and structure of ADRC. So the parameters are independently set according to the principle of separation. The design of the auto disturbance rejection controller is simplified [23]. Among them, for the selection of the nonlinear parameter, $\beta_{1}, \beta_{2}, \beta_{01}, \beta_{02}, \beta_{03}$, an enhanced fireworks algorithm is adopted. In order to obtain satisfactory control performance, the requirements of system speed, stability, accuracy and control quantity are considered comprehensively in this paper. The ITME criterion is selected as the fitness function to evaluate the system performance. The enhanced fireworks algorithm is used to optimize the parameters of ADRC. It avoids the complicated trial and error times of ADRC in the actual debugging process. Forward speed control is taken as an example, the flowchart of the ADRC controller based on the enhanced fireworks algorithm constructed in this paper is shown in Fig.5.

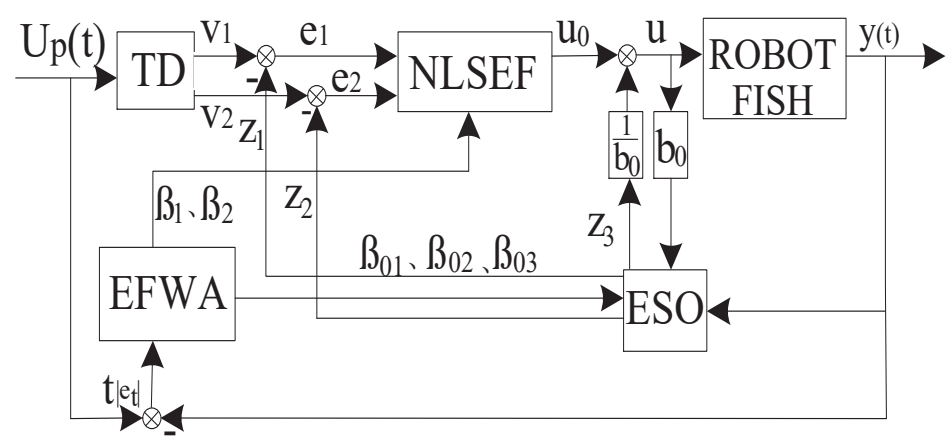

Figure 4: Structure diagram of forward speed controller based on ARDC

In Fig. $4, U_{p(t)}$ is the input signal (the desired speed of the robot fish). $v_{1}(t)$ is the transition process for extracting the $U_{p(t)}$ signal from TD. And $v_{2}$ is the differential signal obtained through. $y(t)$ is the output signal of the controlled object. $z_{1}, z_{2}$ are the state variables of the controlled object of ESO real-time estimation. $z_{3}$ is the disturbance of the controlled object estimated by ESO in real time. $e_{1}, e_{2}$ is the error signal corresponding to $v_{1}, v_{2}$ respectively. $u_{0}(t)$ is the initial control quantity of the controlled object obtained by NLSEF. $u(t)$ is the final control quantity after real-time compensation disturbance. And $b$ is the compensation factor. The $u(t)$ and $y(t)$ subtractive, then absolute errors are obtained, multiplied by the time t to construct the fitness function. After EFWA optimization calculation, the nonlinear parameters $\beta_{1}, \beta_{2}, \beta_{01}, \beta_{02}, \beta_{03}$ are adjusted online to optimize the performance of the system.

The design of the steering speed controller is similar to the design method of the forward speed controller. The paper purpose is to find the optimal control rate $F_{u}$ and tau through the ADRC controller. So the tangential and normal errors are tended to zero. Thereby the robot fish converges to the desired path. Fig. 5 is a flow chart based on the WA-ADRC robotic fish path tracking controller. The desired error information $\tau_{e}, \eta_{e}, \psi_{e}$ can be solved by the desired path information, the real-time position of the robot fish. And the desired control rate $U_{p}, r_{d}$ can be generated from the error information $\tau_{e}, \eta_{e}, \psi_{e}$. The control rates $U_{p}, r_{d}$ are input to the forward speed and steering speed controllers respectively, and the control information $F_{u}$ and tau are generated by the controller. Considering that the robotic fish is an under-actuated model, the lateral input of the robot fish is $F_{v}=0$. And the disturbance is input into the robot fish. After the path generator, the robot fish real-time position is finally obtained. It is obtained the second-order EFWA-ADRC path tracking controller, so that the problem of the position error 


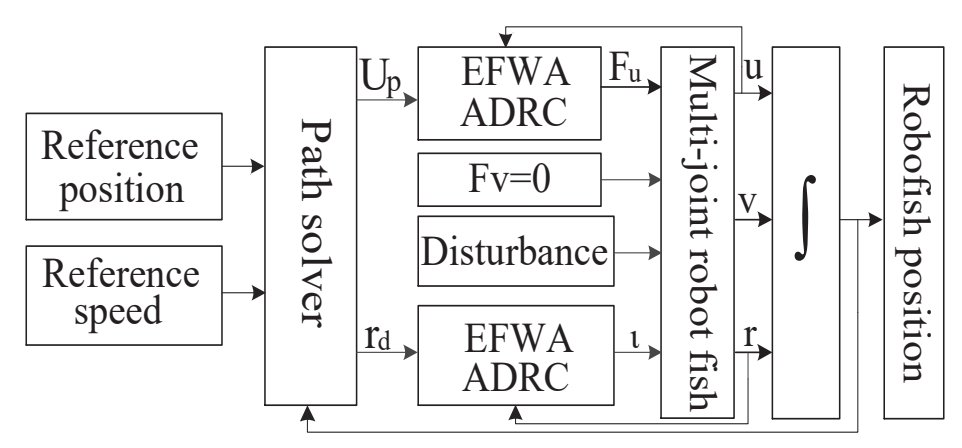

Figure 5: Structure diagram of EFWA-ADRC path tracking controller

stabilization of the robot fish is converted into the stabilization problem of the speed and the heading.

\section{Simulation analysis and experimental verification}

The quality of the robot fish in the paper is $M=8 \mathrm{~kg}$, full length is $I_{B}=0.640 \mathrm{~m}$, the length of trunk is $0.440 \mathrm{~m}$, the length of the stalk is $0.080 \mathrm{~m}$, the length of the caudal fin is $0.120 \mathrm{~m}, I_{x}=$ $0.1095 \mathrm{kgm}^{2}, I_{y}=0.4637 \mathrm{kgm}^{2}, I_{z}=0.4472 \mathrm{kgm}^{2}$, the viscosity coefficient of water movement is $v=1 \times 10^{6} \mathrm{~m}^{2} / \mathrm{s}$. In order to verify the performance of the self-disturbance path tracking controller proposed in the paper, according to the model of the above-mentioned robot fish. The robot fish path tracking simulation system is built in the SIMULINK environment. The linear and circular tracking tasks were used to design simulation experiments and tracking experiments to verify the performance of the path tracking designed and compare it with the conventional ADRC and PID control methods.

\subsection{Simulation analysis of linear tracking}

(1) Simulation analysis of linear tracking Give the linear parameter equation that the robot fish expects to track as equation (23):

$$
\left\{\begin{array}{l}
\xi_{\mathrm{p}}(s)=\sin \theta \\
\eta_{p}(s)=\cos \theta
\end{array}\right.
$$

Where $\theta=\frac{\pi}{4}$ is the angle between the straight path and the x-axis. The initial value of the reference path is $(0,0)$. The expected speed is $U_{d}=1 \mathrm{~m} / \mathrm{s}$. The initial position of the robot fish is $(0,0), \psi=0$. The initial speed is 0 .

Fig.6 shows that the tracking system designed has stability in the tracking task of the straight path. And all three controllers complete the tracking task. It can be seen from Fig.7 that the enhanced fireworks algorithm basically finds the global optimal value after 60 iterations. The fitness function is basically maintained at about 10. In terms of speed control of the robot fish, it can be seen the forward speed and steering speed of the EFWA-ADRC controlled robot can reach the set value in $3 s$, the ADRC is $3.2 s$ in Fig.8 (a) and (b), and in contrast, PID control converges slowly and stays near the expected value at $4 s$. It can be seen the time taken by the three control methods of EFWA-ADRC, ADRC, and PID is $3 s$, and $4 s$ respectively in order to keep the position error of the robot fish positive and negative errors of $0.1 \mathrm{~m}$ from Fig.9 (a), (b), and (c). From Fig. 6 to Fig. 9, it can be seen that the controller based on EFWA-ADRC has 


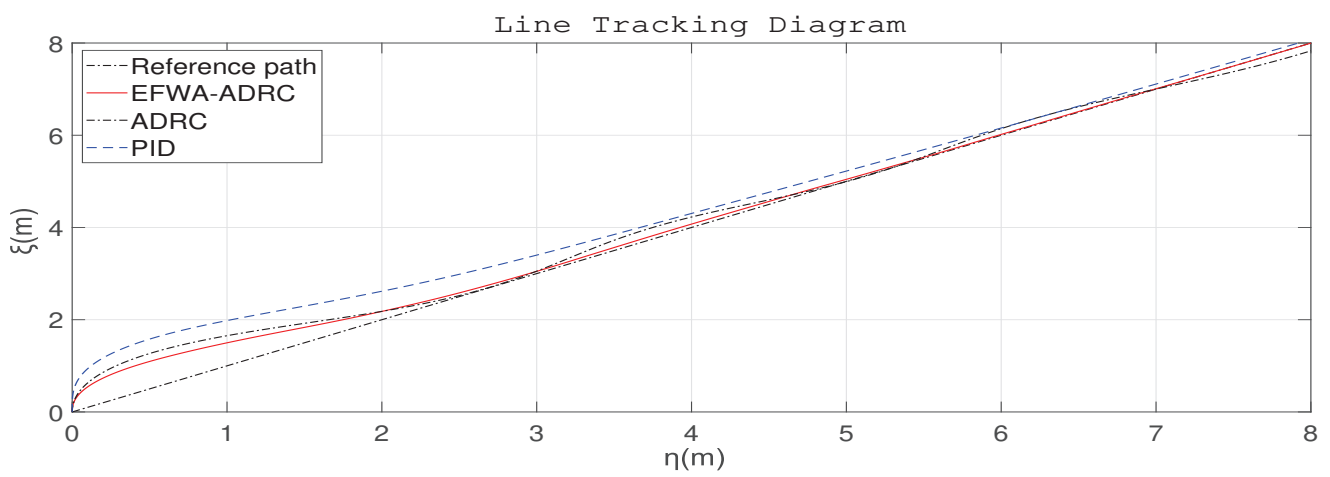

Figure 6: Curve path trace diagram of horizontal plane

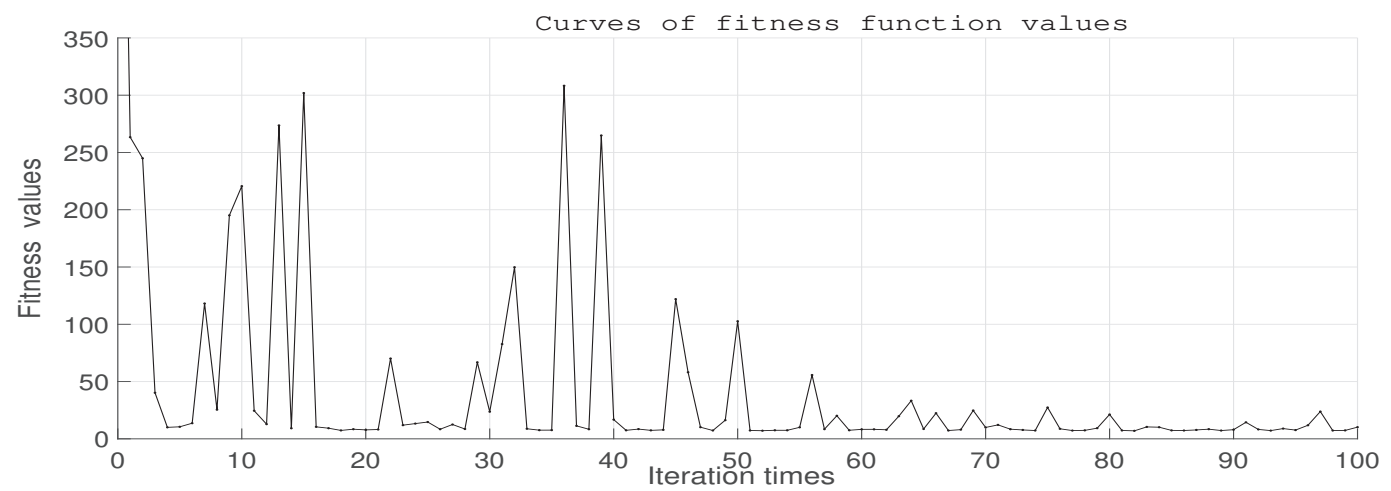

Figure 7: Fitness function curves

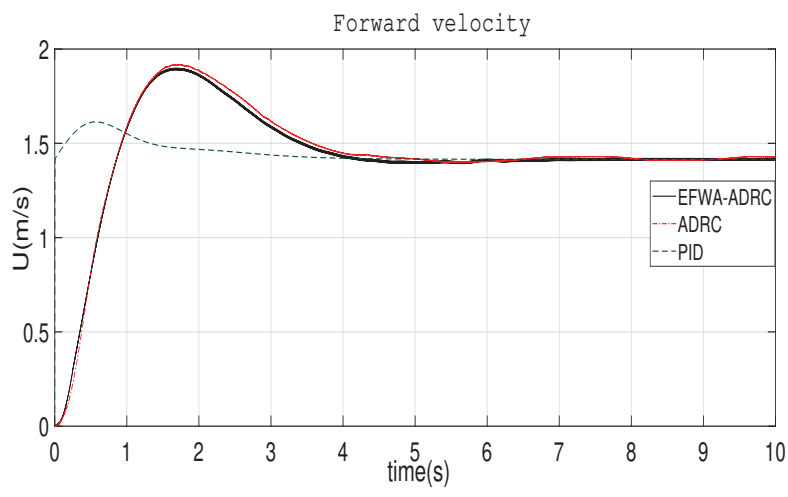

(a) Forword speed.

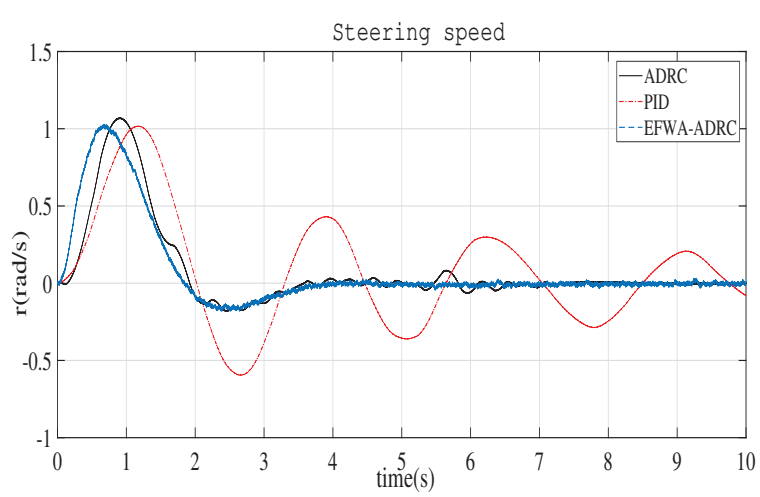

(b)Steering speed.

Figure 8: Robot fish speed curves

short transition time, small overshoot and good anti-interference performance during tracking. Compared with the ADRC control method, the speed response time and position error of the robot fish have been improved, and the control accuracy has been improved by $10 \%$. Therefore, the path tracking controller based on EFWA-ADRC has better dynamic performance and static performance in the linear tracking process is proved.

(2) Curve path tracking simulation analysis It is $\Gamma(s)$ the parameter equation for the robotic fish to track the curve path. 


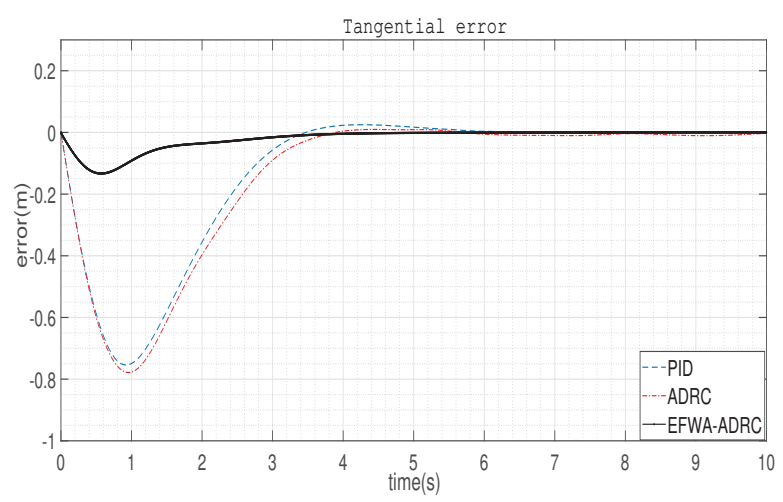

(a) Tangential error.

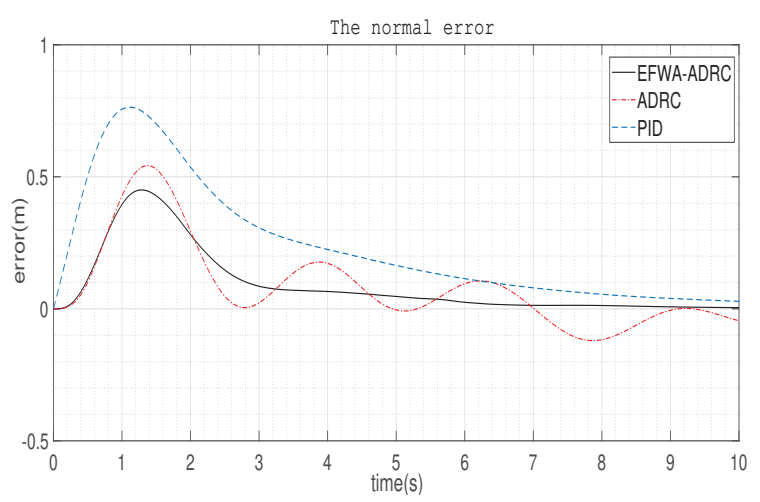

(b)Normal error.

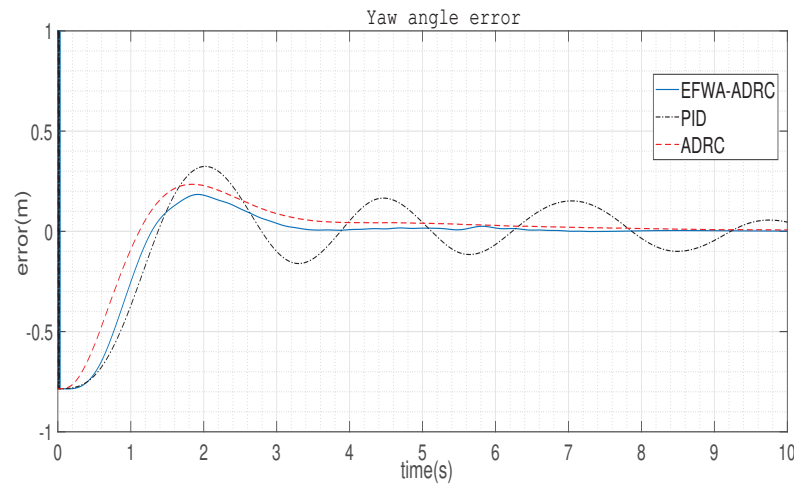

(c) $\psi$ angular error.

Figure 9: Straight line tracking error curves

$$
\left\{\begin{array}{l}
\xi_{p}(s)=\cos (s) \\
\eta_{p}(s)=\sin (s)
\end{array}\right.
$$

The initial value of the reference curve path is $(0,0)$. A circle with a radius of $1 m$, clockwise rotation, the expected speed is $U_{d}=1 \mathrm{~m} / \mathrm{s}$. The initial position of the robot fish is $(0,0) . \psi=0$, the initial speed is0. The simulation results are shown in Fig.10 to Fig.14.

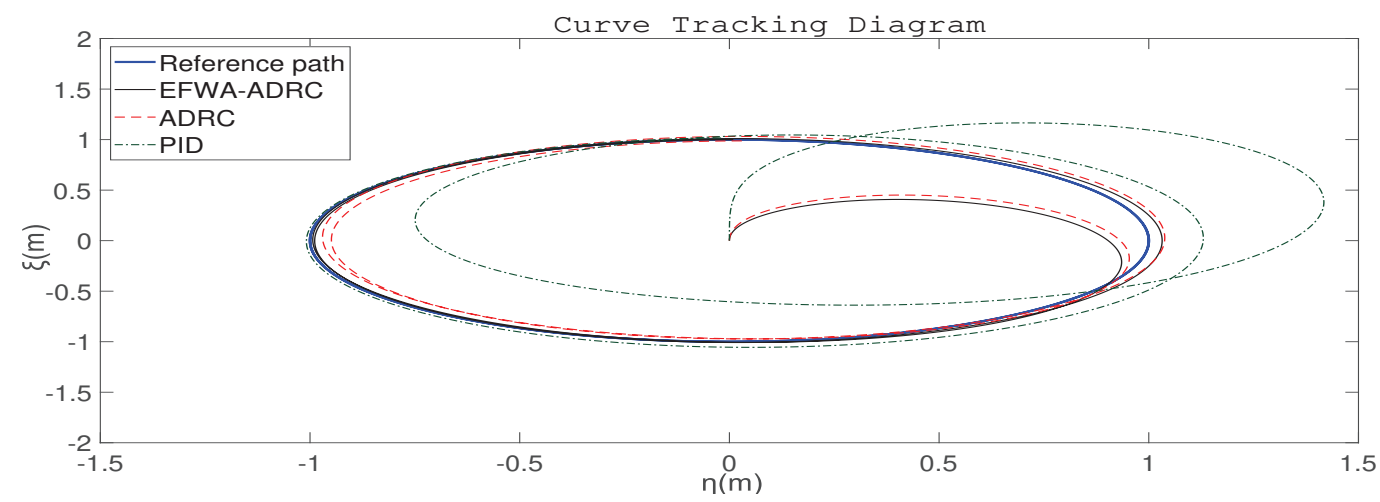

Figure 10: Curve path trace diagram of horizontal plane

It can be seen from Fig. 10 that the ADRC controller has strong robust performance. The tracking system designed also has good stability to the curve tracking task. It can make the 


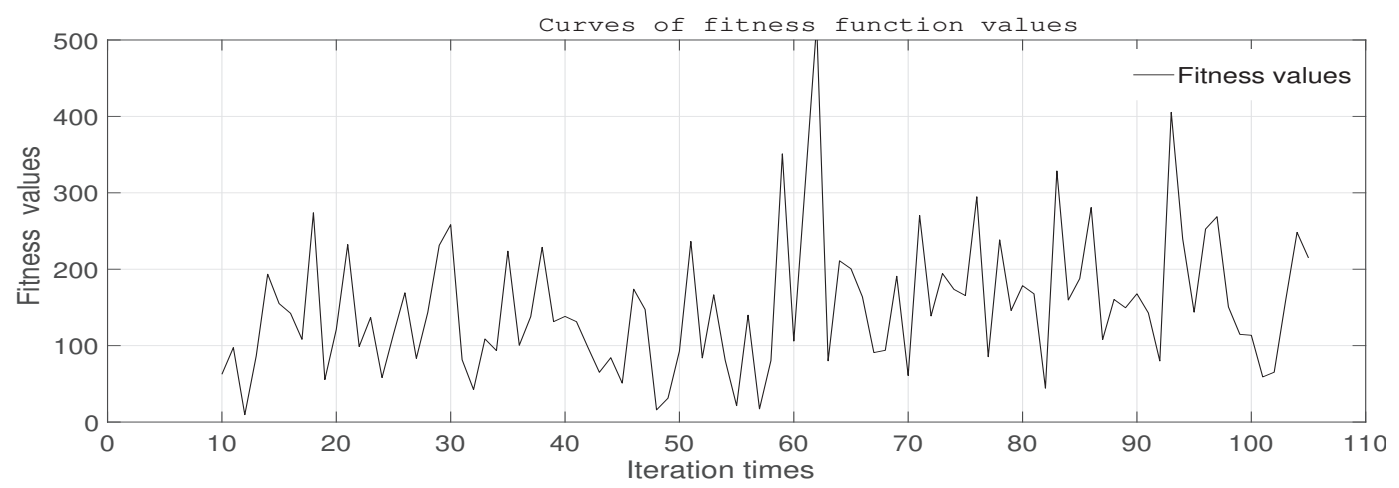

Figure 11: Fitness function curves

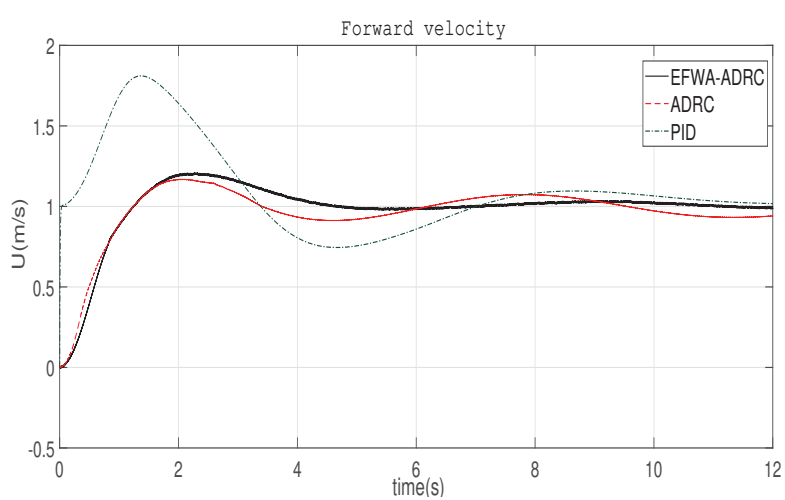

(a) Forword speed.

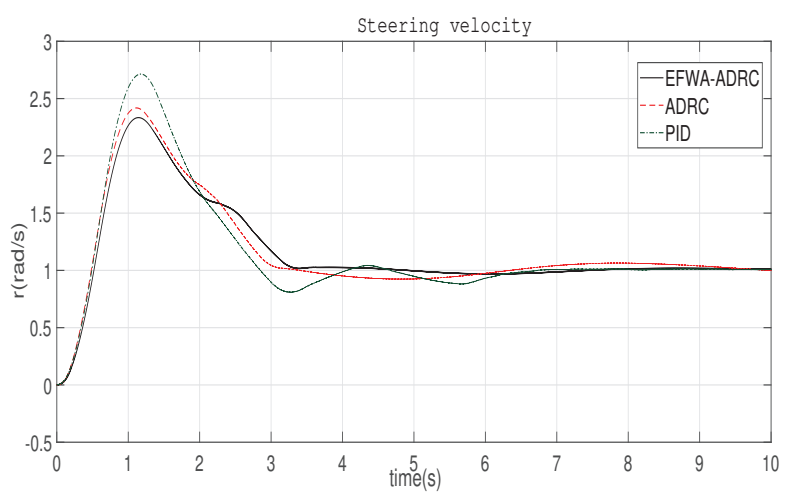

(b)Steering speed.

Figure 12: Robot fish speed curves

robot fish converge to the desired path. In Fig.11 the optimal value of the fitness function of the system reaches 5.21. It can be seen from Fig. 12 that the forward speed and steering speed of the EFWA-ADRC controlled robot can reach the set value in $3 s$, the ADRC is $3.5 s$, and the PID method converges slowly, about 4s. It can be seen from Fig. 13 that the time taken by the three control methods of EFWA-ADRC, ADRC, and PID is $2.8 s, 3 s$, and $4 s$ respectively in order to keep the position error of the robot fish positive and negative errors of $0.1 \mathrm{~m}$. It can be concluded from Fig. 10 to Fig. 13 that the fastness and robustness based on the EFWA-ADRC controller are satisfactory.

Based on the above simulation experiments, it is proposed that the self-disturbance path tracking controller has good control accuracy. Compared with the conventional ADRC controller, the EFWA-ADRC control method has good control effect in terms of accuracy and response time, and it has high control quality and control precision.

\subsection{Experimental verification}

The experimental experiments were carried out on the underwater experimental platform to verify the feasibility of the proposed method. The four-joint robot fish used in the experiment. The length is $0.64 \mathrm{~m}$ and the weight is $8 \mathrm{~kg}$. The size of the fish pond is and the mass of the robot fish is $8 K g$.

(1) Straight path tracking experiment verification The initial position of the robot fish is $(10,5), \psi=0$ the initial speed is $0 \mathrm{~m} / \mathrm{s}$, the reference path is a straight line from $(0,0)$ to $(300,200)$, 


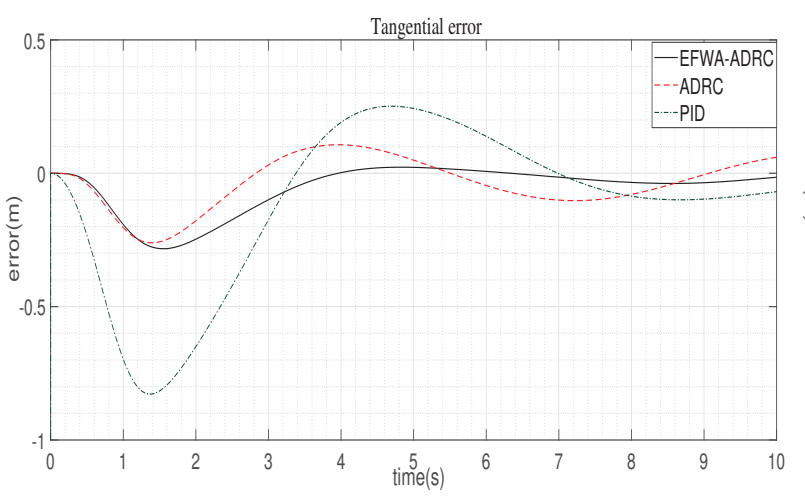

(a) Tangential error.

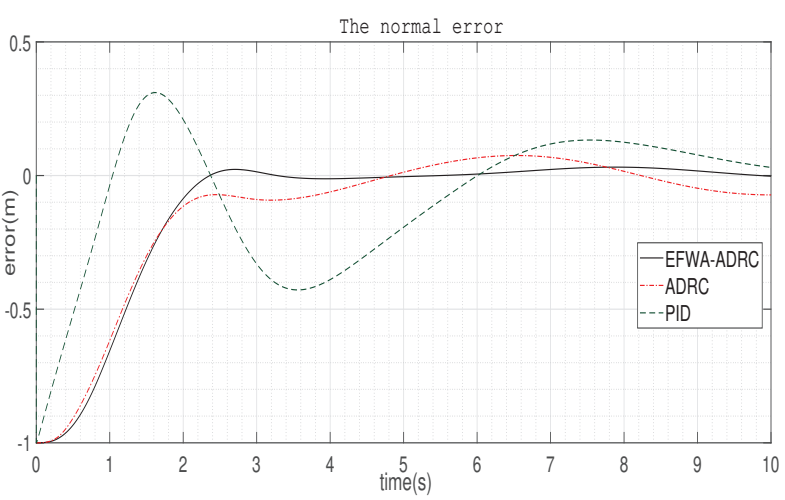

(b)Normal error.

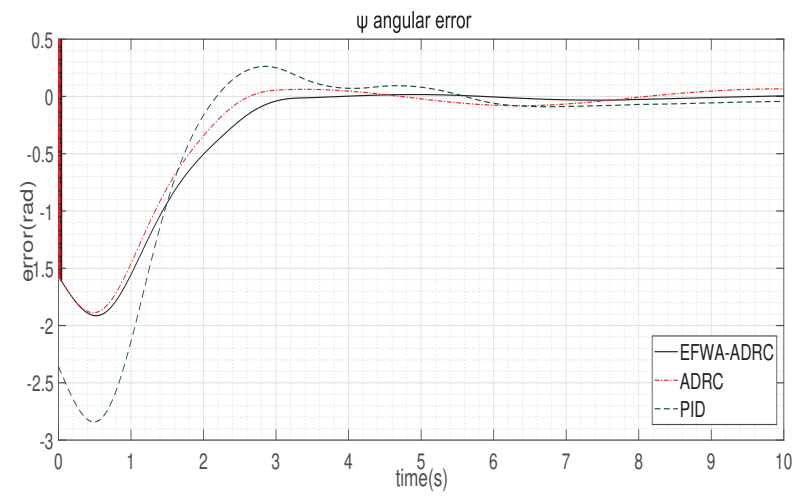

(c) $\psi$ angular error.

Figure 13: Curve tracking error curves

the expected speed of the robotic fish in the experiment is $1 \mathrm{~m} / \mathrm{s}$. it showed in the fig.14- fig.18 the linear path tracking experiment process. In order to reduce the complexity of the debugging process, the ADRC path tracking method with optimized parameters and the tracking method based on the traditional PID are compared. is the experimental picture is showed in the fig.14 based on a conventional PID control method. Fig.15 is an experimental diagram based on the EFWA-ADRC path tracking method.

(2) Curves path tracking experiment verification

In the curve path tracking experiment, the robot fish initial position is $(150,120), \psi=0$. The initial speed is $0 \mathrm{~m} / \mathrm{s}$. the expected speed is $1 \mathrm{~m} / \mathrm{s}$. The reference path is a circle with a center of $(150,120)$ and a radius of $50 \mathrm{~cm}$. The starting point of the reference path is $(200,170)$. The direction angle is $\psi=\pi / 2$. Fig.16 is an experimental diagram based on a conventional PID control method. Fig.17 is an experimental diagram based on the EFWA-ADRC path tracking method. Fig.18 (a) shows the positional trajectory of the robot fish during the linear path tracking process. The yellow line represents the robotic trajectory of the traditional PID control method, and the red one represents the EFWA-ADRC control robotic trajectory. Fig.18(b) is the trajectory during the curve path tracking process, and the desired path represented by black in the two figures. 


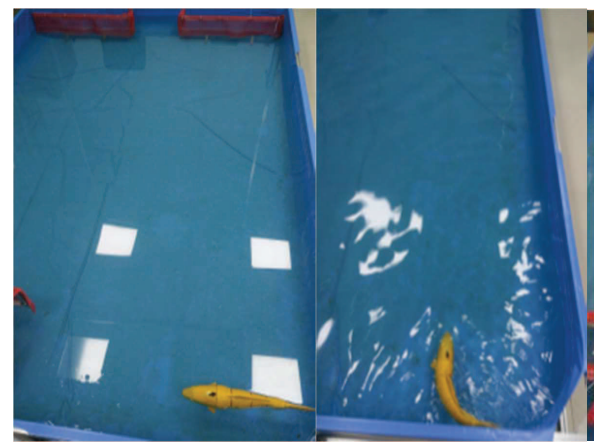

(a) (b)

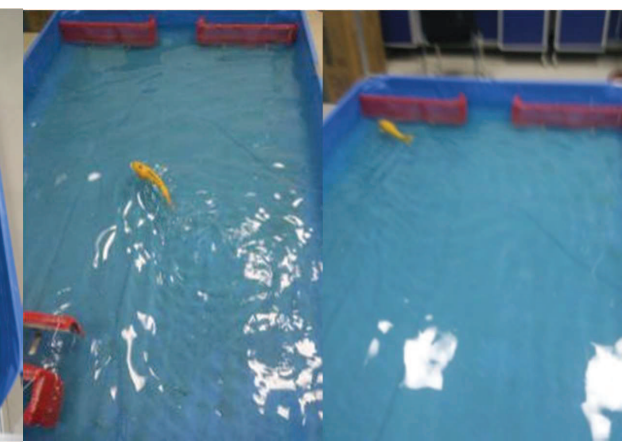

(c) (d)

Figure 14: Experimental pictures based on traditional PID control method

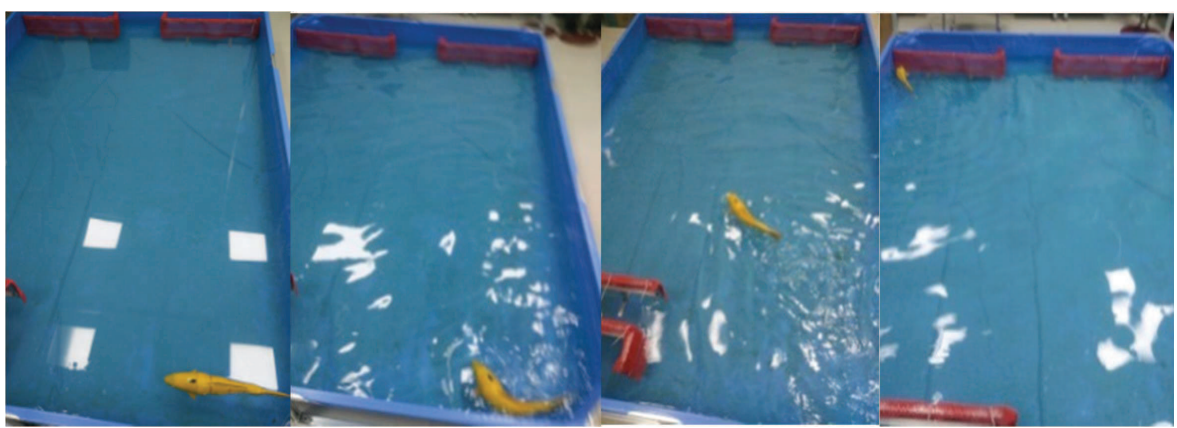

(e) (f) (g)

Figure 15: Experimental pictures based on the EFWA-ADRC path tracking method

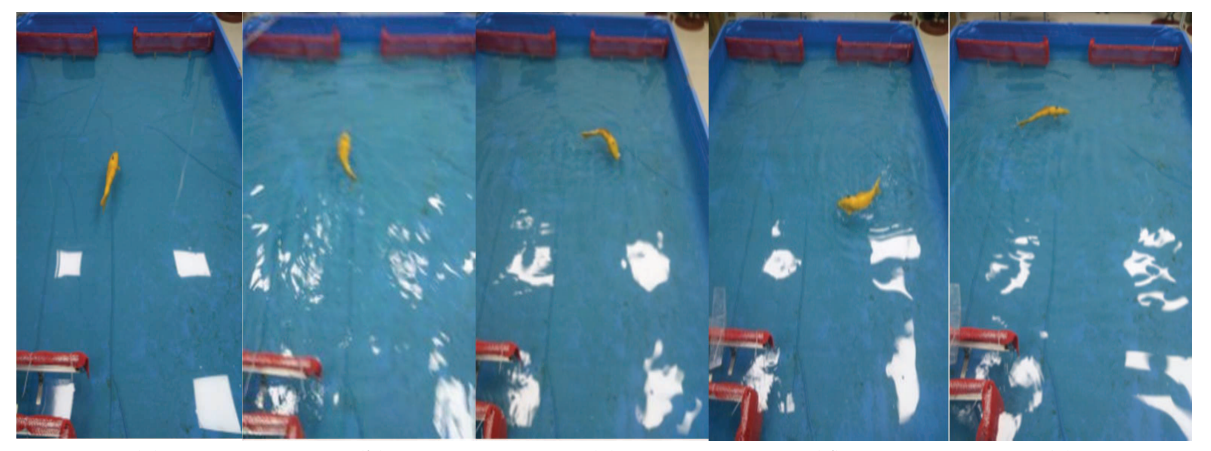

(a)

(b)

(c)

(d)

(e)

Figure 16: Experimental pictures based on traditional PID control method 


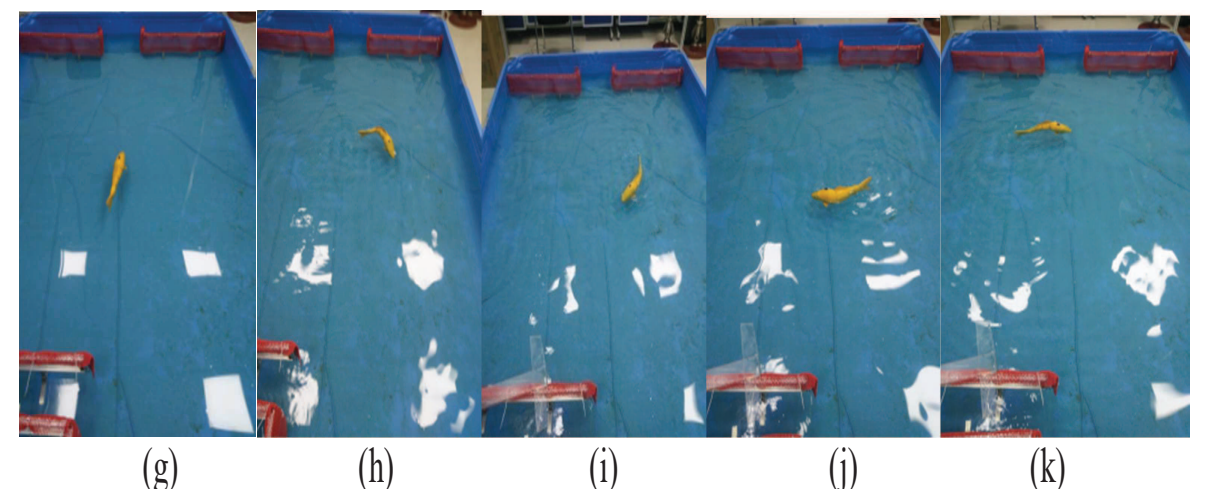

Figure 17: Experimental pictures based on the EFWA-ADRC path tracking method

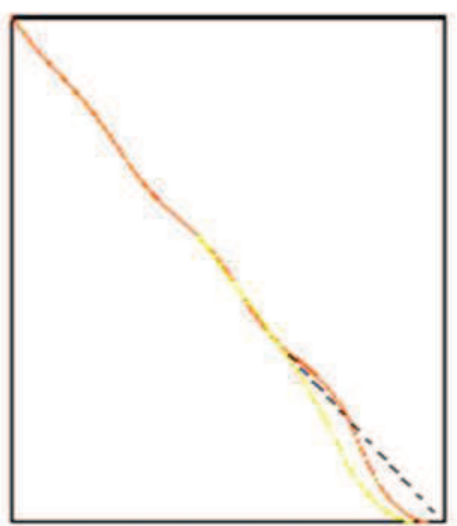

(a)

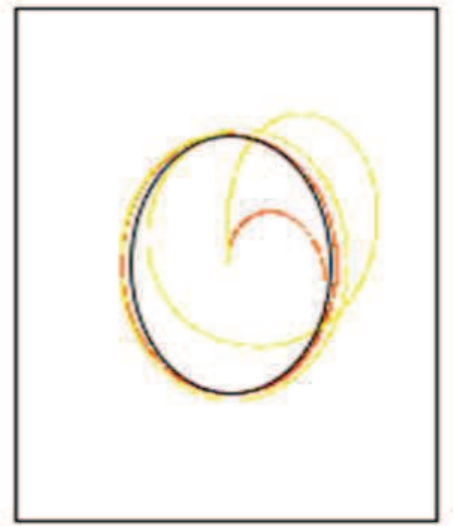

(b)

Figure 18: The experiment trajectories of the robotic fish 
The yellow trajectory in Fig.18 shows that the robot fish trajectory is highly volatile due to the interference of water waves. There is no transition process in the PID control method. It results in a large overshoot of the speed control, and the position error fluctuates greatly. In the ADRC controller the transition process is arranged. So the robot fish speed changes are relatively flat. The unknown disturbance can be estimated and compensated. The red trajectory also proves that the ADRC controller has good anti-interference performance. Therefore, from the combination of simulation and experimental results, the feasibility of the proposed method is proved. The control system has better control precision.

\section{Conclusion}

The robot fish path tracking problem is mainly studied in the paper. The robotic fish path tracking controller is proposed based on the EFWA-ADRC. The virtual mobile robot is introduced combining the kinematics and dynamics equations of multi-joint robotic fish. The tracking error model of the curve path is established in the Serret-Frenet coordinate system. The guidance function of forward and steering control is designed. The tracking errors were reduced by adjusting path parameters and corner velocity. The second-order path controller based on EFWA-ADRC is established. The control information $\mathrm{f}$ and ? are generated. Ultimately the robotic fish can track a given route at a certain speed. Moreover the ADRC parameters are optimized by the fireworks algorithm. So the control precision is further improved. Through simulation analysis and experiment, the feasibility of the proposed method is verified. It has good adaptability to systems with interference and uncertainty. It is further proved that the EFWA-ADRC path tracking controller designed has good control quality in the paper.

\section{Funding}

This project is grateful for the following projects: National Key R\&D Program (2016YFE0111900), Shaanxi International Science and Technology Cooperation Program (2018KW-022), Shaanxi Natural Science Foundation (2018JQ5009 and 18JK0388), and the independent intelligent control research and innovation team support this topic.

\section{Conflict of interest}

The authors declare no conflict of interest.

\section{Bibliography}

[1] Bak, J.; Nguyen, H.N.; Park, S.; Lee, D.; Seo, T.W.; Jin, S.; Kim, J. (2017). Positioning control of an underwater robot with tilting thrusters via decomposition of thrust vector, International Journal of Control Automation ES Systems, 15(5), 1-9, 2017.

[2] Barrett, D.S.; Triantafyllou, M.S., Yue, D.K.P.; Grosenbaugh, M.; Wolfgang, M.J. (1999). Drag reduction in fish-like locomotion, Journal of Fluid Mechanics, 192(392), 183-212, 1999.

[3] Bian, X.Q.; Cheng, X.Q.; Jia, H.M.; Yan, Z.P.; Zhang, L.J. (2011). A bottom-following controller for underactuated AUV based on iterative sliding and increment feedback, Control \& Decision, 26(2): 289-292, 296, 2011. 
[4] Caharija, W.; Pettersen, K.Y.; Bibuli, M.; Calado, P.; Zereik, E.; Braga, J.; Gravdahl, J.T.; Sorensen, A.; Milovanovic, M.; Bruzzone, G. (2016). Integral Line-of-Sight Guidance and Control of Underactuated Marine Vehicles: Theory, Simulations, and Experiments, IEEE Transactions on Control Systems Technology, 24(5), 1623-1642, 2016.

[5] Chao, D.; Yin, Z.; Zhang, Y.; Liu, J.; Sun, X.D.; Zhong, Y.R. (2019). Research on Active Disturbance Rejection Control of Induction Motors Based on Adaptive Particle Swarm Optimization Algorithm with Dynamic Inertia Weight, IEEE Transactions on Power Electronics, 34(3), 2841-1855, 2019.

[6] Fan, S.; Li, B.; Xu, W.; Xu, Y. (2018). Impact of Current Disturbances on AUV Docking: Model-Based Motion Prediction and Countering Approaches. IEEE Journal of Oceanic Engineering, 43(4), 888-904, 2018.

[7] Fossen, T.I.; Lekkas, A.M.(2017). Direct and indirect adaptive integral line-of-sight pathfollowing controllers for marine craft exposed to ocean currents. International Journal of Adaptive Control \& Signal Processing, 31(4), 445-463, 2017.

[8] Gao, C.; Yuan, J.; Zhao, Y. (2018). ADRC for spacecraft attitude and position synchronization in libration point orbits. Acta Astronautica, 145, 238-249, 2018.

[9] Haga, M.; Uchida, M. (2017). Propulsion modeling of caudal fin driving system on balloon fish robot, Artificial Life \& Robotics, 22(1), 10-16, 2017.

[10] Han, J.Q. (1998). Auto-disturbance Controller and Its Application, Control and Decision, 1998(1), 19-23, 1998.

[11] Jung, S. (2018). Improvement of Tracking Control of a Sliding Mode Controller for Robot Manipulators by a Neural Network, International Journal of Control Automation \& Systems, IEEE Int. Conf. on Robotics and Automation, 16(2), 937-943, 2018.

[12] Kelasidi, E.; Liljeback, P.; Pettersen, K.Y. et al(2017). Integral Line-of-Sight Guidance for Path Following Control of Underwater Snake Robots: Theory and Experiments, IEEE Transactions on Robotics, 33(3), 610-628, 2017.

[13] Miller, T.F. (2018). A bio-inspired climb and glide energy utilization strategy for undersea vehicle transit, Ocean Engineering, 149, 78-94, 2018.

[14] Peng, Y.Y.; Yi, G. (2018). Research on Exploring the Patients? Hiding Disease Based on Symptom Weighted Clustering Technique. International Journal Bioautomation, 22(3), 263-274, 2018.

[15] Song, X.R. (2018). A New Hybrid Method in Global Dynamic Path Planning of Mobile Robot, International Journal of Computers Communications \& Control, 13(6), 1032-1046, 2018.

[16] Song, X.; Chen, H.; Xue, Y. (2015). Stabilization precision control methods of photoelectric aim-stabilized system, Optics Communications, 351, 115-120, 2015.

[17] Song, X.R.; Gao, S.; Chen, C.B. (2018). A Novel Vehicle Feature Extraction Algorithm. Traitement Du Signal, 35(3-4), 223-242, 2018.

[18] Tan, Y.; Zhu, Y. (2010). Fireworks Algorithm for Optimization, Advances in Swarm Intelligence. Springer Berlin Heidelberg, 355-364, 2010. 
[19] Wu, L.; Bao, H.; Du, J.L.; Wang, C.S. (2014). A learning algorithm for parameters of automatic disturbances rejection controller. Acta Automatica Sinica, 40(3), 556-560, 2014.

[20] Xiao, Q.; Wang, Y.; Wang, H.(2015). Motion retrieval using weighted graph matching. Soft Computing, 19(1), 133-144,2015.

[21] Xiao, Q.K.; Liu, S.Q. (2017). Motion retrieval based on Dynamic Bayesian Network and Canonical Time Warping, Soft Computing, 21(1), 267-280, 2017.

[22] Xue, P.; Jiang, C.H.; Wei, W.; Lin, J. (2018). Optimization of the intelligent workshop control based on the improved group leadership optimization algorithm. International Journal of Simulation Modelling,, 17(4), 690-702, 2018.

[23] Yang, J.; Ding, Z.; Chen, W.H.; Li, S.H. (2016). Output-based disturbance rejection control for non-linear uncertain systems with unknown frequency disturbances using an observer backstepping approach, Iet Control Theory \& Applications, 10(9), 1052-1060, 2016.

[24] Yen, W.K.; Sierra, D.M.; Guo, J. (2018). Controlling a Robotic Fish to Swim Along a Wall Using Hydrodynamic Pressure Feedback, IEEE Journal of Oceanic Engineering, 43(2), 369$380,2018$.

[25] Zhou, X.; Gao, H.; Zhao, B.; Zhao, L. (2018). A GA-based parameters tuning method for an ADRC controller of ISP for aerial remote sensing applications, ISA Transactions, 81, 318-328, 2018.

[26] Zhang, G.C. ; Huang, H.; Qin, H.D. et al(2017). A novel adaptive second order sliding mode path following control for a portable AUV, Ocean Engineering, 151, 82-92,2018. 